\title{
EFFICACY OF RECOMBINANT EPIDERMAL GROWTH FACTOR IN THE HEALING PROCESS OF DIABETIC ULCER
}

Antony Prabakar ${ }^{1}$, Rasool Syed Raj ${ }^{2}$

${ }^{1}$ Associate Professor, Department of General Surgery, Thanjavur Medical College, Thanjavur.

${ }^{2}$ Assistant Professor, Department of General Surgery, Thanjavur Medical College, Thanjavur.

ABSTRACT
OBJECTIVES
- To evaluate the efficacy of the Recombinant Epidermal Growth Factor in the healing of diabetic ulcer.
- To assess the tolerability of the Recombinant Epidermal Growth Factor in the diabetic ulcer management.

\section{METHODS}

Study Design: Randomized, interventional, prospective, comparative study.

Study Centre: Department of General Surgery and Department of Diabetology, Thanjavur Medical College Hospital, Thanjavur.

Study Period: February 2014-August 2015.

Duration of Study: 18 Months.

Sample Size: 60 (30+30), Study group A-30, Study group B-30.

Sample Drug: Recombinant Epidermal Growth Factor.

\section{Selection Criteria.}

\section{Inclusion Criteria}

1. Age - 20-70 years. 2. Both sexes. 3. Fasting blood sugar $<130 \mathrm{mgs} / \mathrm{dL}$ and postprandial blood sugar $>130 \mathrm{mgs} / \mathrm{dL}<180 \mathrm{mgs} / \mathrm{dL}$ with treatment. 4. Patients with Grade 2 diabetic ulcer. 5. Patients with diabetes along with comorbidities like hypertension with BP $<140 / 90 \mathrm{mmHg}$ with antihypertensives, anaemia with haemoglobin between 8-10 gms\%, chronic renal failure Stage 1 and 2, CAHD patients on drugs with no symptoms at present. 6. Palpable peripheral pulses or Doppler showing flow in peripheral vessels. 7. Patients who give consent and are willing for regular followup.

\section{Exclusion Criteria}

1. Patients with extensive gangrenous changes. 2. Patients with vascular occlusion (Absent peripheral pulses or no flow in peripheral vessels in the Doppler study). 3. Patients with diabetic ketoacidosis or with fasting blood sugar $>130 \mathrm{mgs} / \mathrm{dL}$, postprandial blood sugar $>180 \mathrm{mgs} / \mathrm{dL}$ in spite of treatment. 4. Patients with diabetes with hypertension with BP $>140 / 90 \mathrm{mmHg}$, anaemia with haemoglobin $<8$ gms\%, chronic renal failure with stage 3, 4, 5 congestive heart failure and recent onset myocardial infarction. 5. Pregnant or breastfeeding women. 6. Not willing for the study or not willing for regular visits.

\section{CONCLUSION}

The conclusion of this study is that Recombinant Epidermal Growth Factor is effective in the healing of diabetic ulcers at significantly higher rate. No adverse reactions are encountered. It is tolerated well by all the patients.

\section{KEYWORDS}

Diabetic Ulcers, Recombinant Growth Factor, Lower Limb Ulcers.

HOW TO CITE THIS ARTICLE: Prabakar A, Raj RS. Efficacy of recombinant epidermal growth factor in the healing process of diabetic ulcer. J. Evolution Med. Dent. Sci. 2016;5(31):1642-1648, DOI: 10.14260/jemds/2016/387

\section{INTRODUCTION}

Wound healing is a process that leads to tissue restoration and normal functioning. It is synonymous with an organism's defence response to injury, which leads to reformation of the organ's defect by the initiation of chain reactions of pathways.

Financial or Other, Competing Interest: None.

Submission 14-02-2016, Peer Review 22-03-2016,

Acceptance 28-03-2016, Published 18-04-2016.

Corresponding Author:

Dr. Antony Prabakar,

No. 24 A, Lakshmi Apartments,

New Colony $1^{\text {st }}$ Street,

VOC Nagar, Anna Nagar East,

Chennai-600102,

Tamilnadu.

E-mail: dr.a.prabakar@gmail.com

DOI: $10.14260 /$ jemds $/ 2016 / 387$
Once a Tissue is Injured, the response to Injury Occurs in Either of the Two Ways.

1. Formation of scar-different cellular matrix filling the defect, or

2. Regeneration of tissue - complete replacement of the original architecture of the tissue.

\section{The Stages in Wound Healing are Sequenced as. ${ }^{1}$}

1. Phase of inflammation.

2. Phase of cellular proliferation.

3. Phase of maturation and remodeling.

Inflammation is initiated by a constellation of factors such as growth factor, proteases, kinins, cytokines, eicosanoids and cellular metabolites. All these factors set in motion the wound stabilization, infection eradication and tissue replacement. All these process depend on the regulation of inflammatory mediators. 
The topic of study, the growth factors or modulating factors, are peptides involved in signalling which are increasingly produced during inflammation and target nonhaematopoietic cells. The factors of prime importance are Fibroblast Growth Factor (FGF), Insulin like Growth Factor (IGF), Platelet Derived Growth Factor (PDGF), Vascular Endothelial Growth Factor (VEGF), Epidermal Growth Factor (EGF) and Transforming Growth Factor beta. They act locally on the target site without systemic side effects.

These healing cycles in inflammation are extremely deranged in longstanding wounds like diabetic foot ulcer, which lead to failure of epithelialization and granulation tissue formation. These failures along with neuropathy and joint instability (Biomechanical) result in debilitating ulcer further exaggerated by arterial insufficiency (30-60\%) and absence of strict glucose regulation.

The wide reaching negative social impact of non-healing chronic diabetic ulcers is emphasized by the estimate that 70 million of the world population might be affected with diabetes by $2025 .^{2}$ and these people will be physically and socially handicapped if they develop foot problems, which demands increased hospitalization and also the fact that chronic diabetic foot ulcers accounts for $20-25 \%$ of all hospital stays.

A novel approach for combating longstanding diabetic wounds is the external application of the growth factors, which hastens healing in impaired models of wound healing.

A vital cog in the wheel of wound healing, Epidermal Growth Factor has profound implication in fibroblast function, keratinocyte migration and formation of granulation tissue. Its earlier limitation of rapid degradation in chronic wound surroundings has been overcome by protein stabilizing drug delivery system.

Hence, this study has been done with the view to evaluate the efficacy of Epidermal Growth Factor in accelerating the healing of diabetic foot ulcers. This study also compares the Epidermal Growth factor vs the standard therapy of wash with antiseptic solution and moist saline dressing in healing of diabetic ulcer disease.

Risk Factors for Ulceration

The below table shows the risk factors in diabetic foot.

\begin{tabular}{|c|c|}
\hline Relative Risk/Odds Ratio & Systemic Risk Factors \\
\hline 1.02 to 2.13 & Hypertension \\
\hline 1.02 to 6.4 & Hyperlipidaemia \\
\hline 1.3 to 3.2 & Hyperglycaemia \\
\hline 2.6 to 5.2 & Male sex \\
\hline 1.07 to 3.7 & Retinopathy \\
\hline 2 & Above 65 years \\
\hline 1.9 & Poor vision \\
\hline 1.2 & Obesity \\
\hline 2.4 & Proteinuria \\
\hline
\end{tabular}

\begin{tabular}{|c|c|}
\hline $\begin{array}{c}\text { Relative Risk/Odds } \\
\text { Ratio }\end{array}$ & Local Risk Factors \\
\hline 1.6 to 18 & History of foot ulcer or amputation \\
\hline 3.3 to 3.5 & Structural foot deformity \\
\hline 2.0 to 5.9 & Abnormal foot pressures \\
\hline 2.2 to 18.4 & Sensory neuropathy \\
\hline 2.4 to 3 & Peripheral vascular disease \\
\hline
\end{tabular}

\section{The Epidermal Growth Factor}

It is discovered by Stanley Cohen and Rita Levi Montalcini. ${ }^{3}$ They won the Nobel Prize in Medicine and Physiology for their discovery. It is a peptide with low molecular weight. It has 53 amino acids. Its molecular weight is $6045 \mathrm{Da}$. It lacks three amino acids namely - lysine, phenylalanine, alanine. ${ }^{4}$ It was first isolated from the submandibular gland of mouse. The Epidermal Growth Factor belongs to a group of family called the EGF family. It also includes TGF-alpha, Betacellulin, Epigen, Epiregulin, Amphiregulin, Heparin binding EGF. The gene coding EGF is located in chromosome. ${ }^{4}$

Prepro EGF is a transmembrane precursor protein with a mature sequence in the extracellular domain. Cleavage of Arg/His and Arg/Asp dipeptide bonds at $\mathrm{N}$ and $\mathrm{C}$ terminal ends resulting in the formation of soluble peptide. ${ }^{5}$ It has two domains.

- $\quad \mathrm{N}$ terminal-major antiparallel beta sheet structures and two of the disulphide bonds.

- C terminal - double hairpin structure, a minor antiparallel beta sheet and the third disulphide bond.

- The gene coding EGF is located in chromosome. ${ }^{4}$

\begin{tabular}{|c|c|c|c|}
\hline Sl. No. & Action of EGF & $\begin{array}{l}\text { Clinical Uses } \\
\end{array}$ & Trial \\
\hline 1. & $\begin{array}{l}\text { Smooth muscles, epithelial cell and } \\
\text { fibroblast proliferation }\end{array}$ & $\begin{array}{l}\text { Wound healing. }{ }^{6} \text { in diabetic ulcer, venous ulcer, healing of burns } \\
\text { wound }\end{array}$ & $\begin{array}{c}\text { Human } \\
\text { trial }\end{array}$ \\
\hline 2. & $\begin{array}{l}\text { Maintains integrity of oesophageal } \\
\text { and gastric mucosa, inhibits gastric } \\
\text { acid secretion, stimulation of DNA } \\
\text { synthesis, mucosal protection. }\end{array}$ & $\begin{array}{l}\text { Healing of duodenal ulcers, also being tried in Zollinger-Ellison } \\
\text { syndrome }\end{array}$ & $\begin{array}{c}\text { Human } \\
\text { trial }\end{array}$ \\
\hline 3. & $\begin{array}{l}\text { Increased proliferation of } \\
\text { duodenal, jejunal cells and } \\
\text { increased transport of nutrients } \\
\text { across the small intestine. }{ }^{7}\end{array}$ & $\begin{array}{l}\text { Intestinal atrophy, Crohn's, after massive enterectomy, also tried } \\
\text { in necrotizing enterocolitis. }\end{array}$ & $\begin{array}{c}\text { Animal } \\
\text { trial }\end{array}$ \\
\hline 4. & $\begin{array}{l}\text { Decreased bacterial translocation } \\
\text { from the gut in acute pancreatitis. }{ }^{7}\end{array}$ & Preventing systemic infection & $\begin{array}{c}\text { Animal } \\
\text { trial }\end{array}$ \\
\hline 5. & $\begin{array}{l}\text { EFGR is over-expressed in high- } \\
\text { grade gliomas and muscle invasive } \\
\text { urothelial cancer of bladder.8 }\end{array}$ & Targeting tumour cells to decreases tumour load & $\begin{array}{c}\text { Animal } \\
\text { trial }\end{array}$ \\
\hline 6. & $\begin{array}{l}\text { Epithelial regeneration in wounded } \\
\text { cornea along with hyaluronic acid. } 8\end{array}$ & Repair of eye damage & $\begin{array}{c}\text { Animal } \\
\text { trial }\end{array}$ \\
\hline 7. & Regeneration of hepatocytes. ${ }^{7}$ & Repair of liver injury & $\begin{array}{c}\text { Animal } \\
\text { trial }\end{array}$ \\
\hline
\end{tabular}




\begin{tabular}{|c|c|c|c|}
\hline 8. & $\begin{array}{c}\text { Closure of tympanic membrane } \\
\text { perforation }\end{array}$ & & $\begin{array}{c}\text { Human } \\
\text { trial }\end{array}$ \\
\hline 9. & $\begin{array}{l}\text { Active sodium transport and } \\
\text { clearance of lung fluid }\end{array}$ & Decreases pulmonary oedema & $\begin{array}{c}\text { Animal } \\
\text { trial }\end{array}$ \\
\hline 10. & $\begin{array}{c}\text { Enhances the sensitivity of many } \\
\text { cancer cells (Ovary, head and neck, } \\
\text { cervix, colon, pancreas, prostate, } \\
\text { lung) to drugs like cisplatin, 5-FU, } \\
\text { melphalan. }{ }^{8}\end{array}$ & Potentiating anti-cancer agents & $\begin{array}{c}\text { Human } \\
\text { trial }\end{array}$ \\
\hline 11. & $\begin{array}{c}\text { Inhibits enteropathogenic E. coli } \\
\text { colonization }\end{array}$ & Traveller's diarrhoea & $\begin{array}{c}\text { Human } \\
\text { trial }\end{array}$ \\
\hline 12. & $\begin{array}{l}\text { Tissue repair and regeneration, } \\
\text { formation of collagen and elastic } \\
\text { fibres. }\end{array}$ & Anti-ageing cream & \\
\hline
\end{tabular}

\section{MECHANISM OF ACTION OF EGF.9,10,11,1}

Mechanism of Action of Egf
Epidermal growth factor + Epidermal growth factor receptor
$\downarrow$
Activation of tyrosine kinase activity
$\downarrow$
Signal transduction cascade
$\downarrow$
Results in increased intracellular calcium, increased
glycolysis increased protein synthesis and increased
expression of certain genes.

\section{Biochemical Changes Occurring at the Cellular Level}

The Epidermal Growth Factor results in the increased transport of aminoisobutyric acid and uridine into cells. The incorporation of uridine into the RNA is also increased. The stimulation of RNA synthesis is also increased.

\section{RESULTS}

1. The rate of healing of ulcers $<5 \mathrm{~cm}$ are compared between the EGF and the control groups. In EGF group is $91.67 \%$ and in the control group is $84.62 \%$.

2. The rate of healing of ulcers $>5 \mathrm{~cm}$ are compared between the two groups. In EGF group is $83.33 \%$ and in control group is $52.94 \%$.

3. Overall, the rate of healing of ulcers in the EGF group are compared with the control group. Rate of healing in EGF group is $86.67 \%$ and control group is $66.67 \%$. Next, the duration of healing of the ulcers are compared between the two groups. Those ulcers which are not healed by 15 weeks and those that are treated by skin grafting are considered as non-healing and are not considered in the T test.

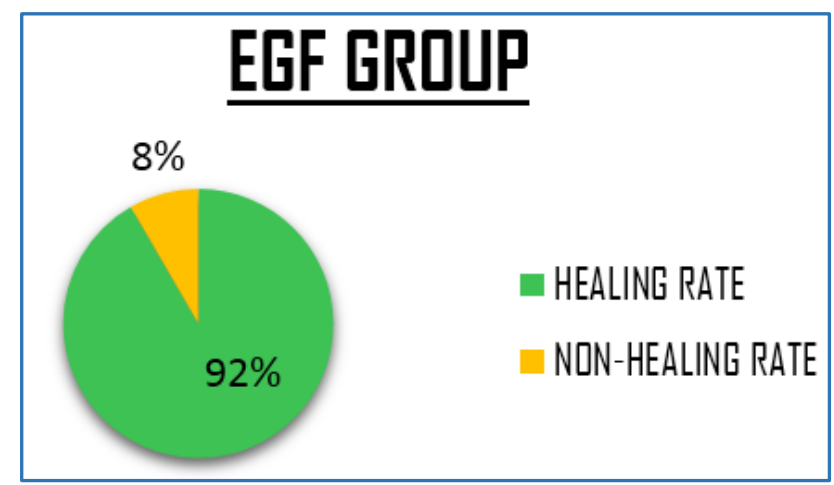

Healing Rate and Non-Healing Rate in EGF Group

\section{CONTROL GROUP}

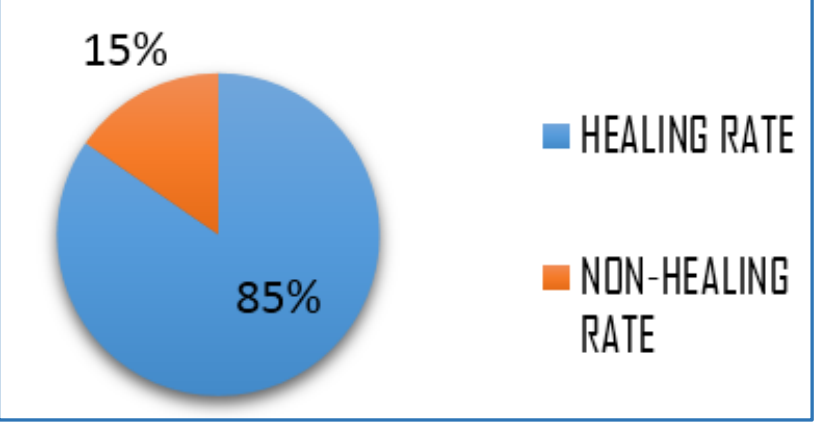
Healing Rate and Non-Healing Rate
in Control Group

\section{DISCUSSION}

The rate of healing of ulcers $<5 \mathrm{~cm}$ in the EGF treated group is significantly greater than in the control group. The rate of healing of ulcers $>5 \mathrm{~cm}$ in the EGF treated group is significantly greater than in the control group. Overall, the rate of healing of ulcers in the EGF treated group is significantly greater than in the control group.

\section{CONCLUSION}

The conclusion of this study is that Recombinant Epidermal Growth Factor is effective in the healing of diabetic ulcers at a significantly higher rate. No adverse reactions are encountered. It is tolerated well by all the patients.

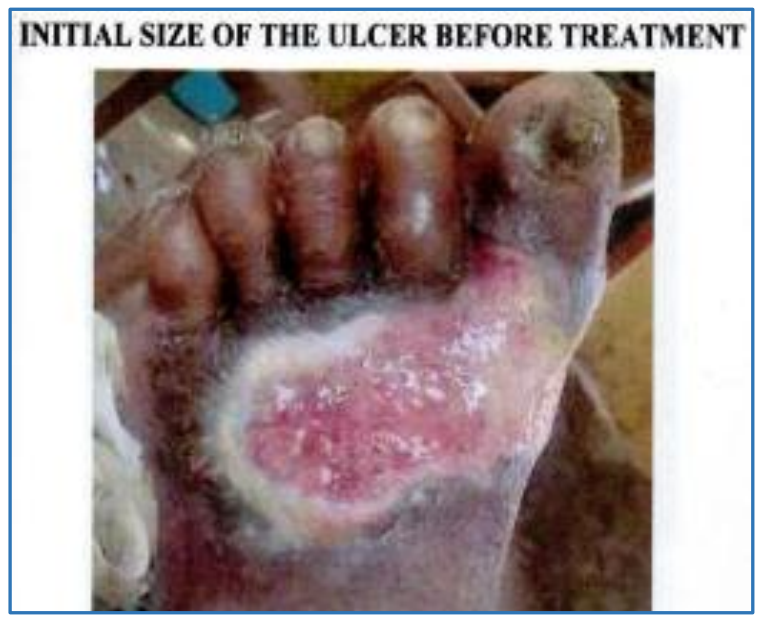



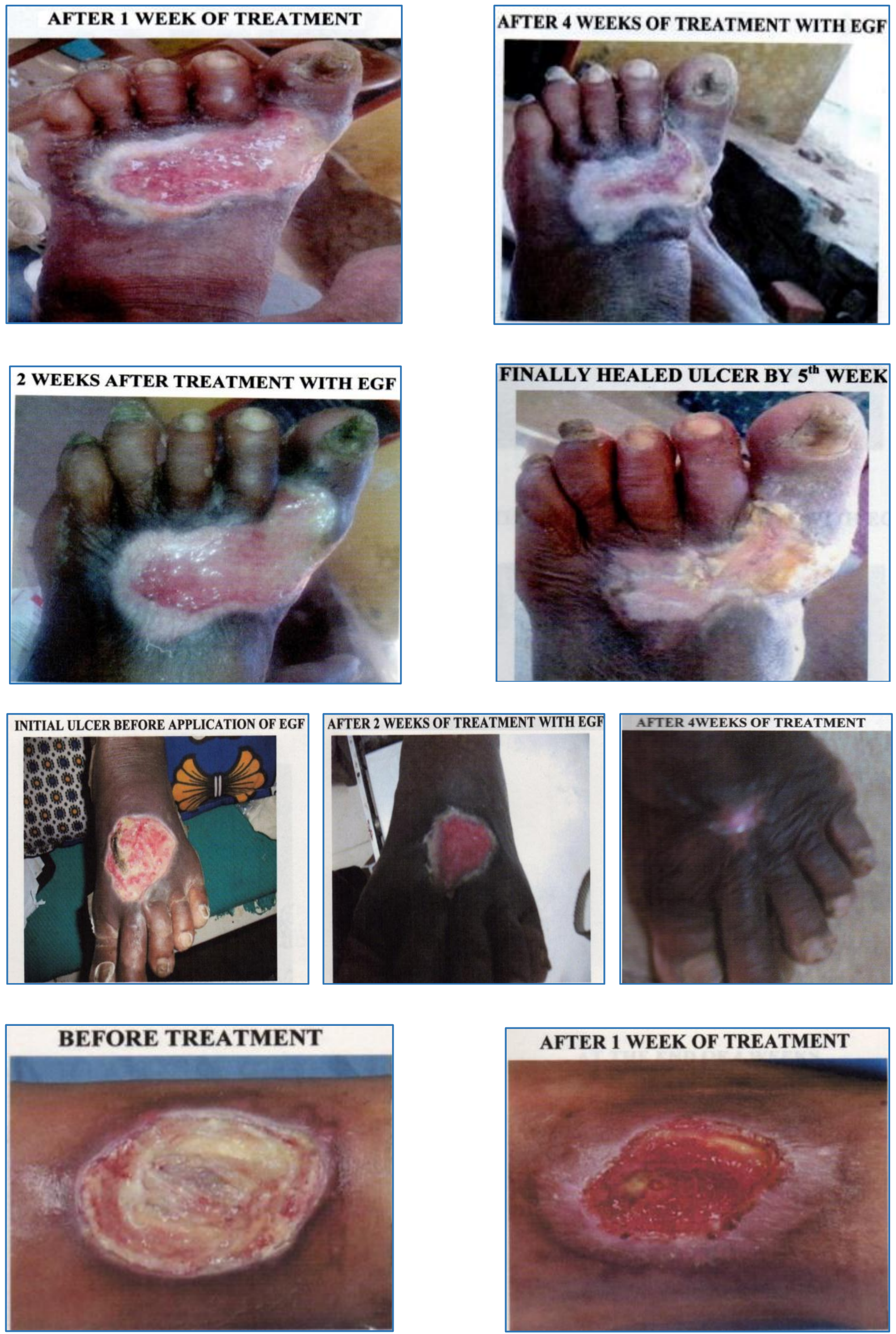


\begin{tabular}{|c|c|c|c|c|c|c|c|c|c|c|}
\hline $\begin{array}{l}\text { Sl. } \\
\text { No. }\end{array}$ & $\begin{array}{l}\text { AGE } \\
\text { /SEX } \\
\end{array}$ & IP NO. & ULCER SITE & DOPPLER & $\begin{array}{l}\text { NEURO- } \\
\text { PATHY }\end{array}$ & $\begin{array}{c}\text { RISK } \\
\text { FACTORS } \\
\end{array}$ & $\begin{array}{c}\text { PUS } \\
\text { CULTURE } \\
\end{array}$ & SENSITIVITY & $\begin{array}{l}\text { HEALED } \\
\text { OR NOT } \\
\end{array}$ & $\begin{array}{c}\text { DURATI } \\
\text { ON } \\
\end{array}$ \\
\hline 1. & $53 / \mathrm{M}$ & 1478345 & $\begin{array}{l}\text { DORSUM OF R } \\
\text { FOOT }\end{array}$ & $\begin{array}{l}\text { MONOPHA } \\
\text { SIC-PT, DP }\end{array}$ & YES & NO & E. COLI, KLE & AMI & $\begin{array}{c}\text { NOT } \\
\text { HEALED }\end{array}$ & - \\
\hline 2. & $30 / \mathrm{M}$ & 1458656 & $\begin{array}{l}\text { DORSUM OF R } \\
\text { FOOT }\end{array}$ & $\begin{array}{l}\text { MONOPHA } \\
\text { SIC FLOW- } \\
\text { DP } \\
\end{array}$ & NO & HT & STAPH & CIPRO & HEALED & $\begin{array}{c}9 \\
\text { WEEKS }\end{array}$ \\
\hline 3. & $38 / F$ & 1383872 & $\begin{array}{l}\text { DORSUM OF L } \\
\text { FOOT }\end{array}$ & $\begin{array}{c}\text { BIPHASIC } \\
\text { FLOW-PT, } \\
\text { DP }\end{array}$ & NO & ANAEMIA & STAPH & CIPRO, AMP & HEALED & $\begin{array}{c}12 \\
\text { WEEKS }\end{array}$ \\
\hline 4. & $54 / \mathrm{M}$ & 1466846 & $\begin{array}{l}\text { DORSUM OF L } \\
\text { FOOT }\end{array}$ & NORMAL & YES & NO & - & - & HEALED & $\begin{array}{c}5 \\
\text { WEEKS } \\
\end{array}$ \\
\hline 5. & $28 / F$ & 1463707 & $\begin{array}{l}\text { PLANTAR R } \\
\text { FOOT }\end{array}$ & $\begin{array}{l}\text { MONOPHA } \\
\text { SIC-PT, DP }\end{array}$ & NO & HT & ANAEROBE & METRO & HEALED & $\begin{array}{c}10 \\
\text { WEEKS }\end{array}$ \\
\hline 6. & $60 / F$ & 1464852 & $\begin{array}{l}\text { DORSUM OF R } \\
\text { FOOT }\end{array}$ & $\begin{array}{l}\text { BIPHASIC- } \\
\text { PA, PT, DP }\end{array}$ & NO & $\begin{array}{c}\text { CRF/ } \\
\text { ANAEMIA }\end{array}$ & - & - & HEALED & $\begin{array}{c}7 \\
\text { WEEKS } \\
\end{array}$ \\
\hline 7. & $38 / \mathrm{M}$ & 1458577 & $\begin{array}{l}\text { DORSUM OF R } \\
\text { FOOT }\end{array}$ & NORMAL & YES & NO & E. COLI & $\mathrm{CE}$ & HEALED & $\begin{array}{c}8 \\
\text { WEEKS } \\
\end{array}$ \\
\hline 8. & $55 / \mathrm{M}$ & 1476472 & $\begin{array}{l}\text { DORSUM OF L } \\
\text { FOOT }\end{array}$ & $\begin{array}{c}\text { MONOPHA } \\
\text { SIC - } \\
\text { PA,PT,DP } \\
\end{array}$ & NO & HT/CAHD & STREP & $P$ & $\begin{array}{l}\text { GRAFTI } \\
\text { NG }\end{array}$ & - \\
\hline 9. & $60 / M$ & 1469248 & $\begin{array}{l}\text { PLANTAR R } \\
\text { FOOT }\end{array}$ & $\begin{array}{l}\text { MONOPHA } \\
\text { SIC-PT,DP }\end{array}$ & NO & CRF & ANAEROBE & METRO & $\begin{array}{c}\text { NOT } \\
\text { HEALED }\end{array}$ & - \\
\hline 10. & $68 / F$ & 1496923 & $\begin{array}{l}\text { DORSUM OF L } \\
\text { FOOT }\end{array}$ & NORMAL & YES & NO & - & - & & \\
\hline 11. & $59 / \mathrm{M}$ & 1478281 & $\begin{array}{l}\text { DORSUM OF R } \\
\text { FOOT }\end{array}$ & NORMAL & NO & CAHD & STREP & CE, AMP & HEALED & $\begin{array}{c}9 \\
\text { WEEKS }\end{array}$ \\
\hline 12. & $55 / \mathrm{M}$ & 1410363 & $\begin{array}{l}\text { DORSUM OF R } \\
\text { FOOT }\end{array}$ & $\begin{array}{c}\text { BIPHASIC } \\
\text { FLOW- } \\
\text { PT,DP } \\
\end{array}$ & NO & $\begin{array}{l}\text { CRF/ANAE } \\
\text { MIA }\end{array}$ & STREP & CE/CIPRO & $\begin{array}{l}\text { GRAFTI } \\
\text { NG }\end{array}$ & - \\
\hline 13. & $52 / \mathrm{M}$ & 1481042 & $\begin{array}{l}\text { DORSUM OF L } \\
\text { FOOT }\end{array}$ & NORMAL & YES & NO & KLEB & AMI, GM & HEALED & $\begin{array}{c}9 \\
\text { WEEKS }\end{array}$ \\
\hline 14. & $57 / F$ & 1448401 & $\begin{array}{l}\text { DORSUM OF R } \\
\text { FOOT }\end{array}$ & $\begin{array}{l}\text { MONOPHA } \\
\text { SIC-PT,DP }\end{array}$ & NO & CAHD & - & - & HEALED & $\begin{array}{c}12 \\
\text { WEEKS }\end{array}$ \\
\hline 15. & $45 / M$ & 1464058 & $\begin{array}{l}\text { DORSUM OF L } \\
\text { FOOT }\end{array}$ & $\begin{array}{c}\text { BIPHASIC } \\
\text { FLOW- } \\
\text { PT,DP } \\
\end{array}$ & YES & CRF & FUSIFORM & CIPRO, AM & HEALED & $\begin{array}{c}7 \\
\text { WEEKS }\end{array}$ \\
\hline 16. & $37 / F$ & 1447369 & $\begin{array}{l}\text { DORSUM OF L } \\
\text { FOOT }\end{array}$ & NORMAL & YES & NO & $\begin{array}{l}\text { E. COLI, } \\
\text { STREP }\end{array}$ & CE, SEPTRAN & HEALED & $\begin{array}{c}6 \\
\text { WEEKS }\end{array}$ \\
\hline 17. & $68 / \mathrm{M}$ & 1473251 & $\begin{array}{c}\text { DORSUM OF R } \\
\text { FOOT }\end{array}$ & $\begin{array}{l}\text { MONOPHA } \\
\text { SIC-PT,DP }\end{array}$ & YES & HT/CAHD & - & - & HEALED & $\begin{array}{c}8 \\
\text { WEEKS }\end{array}$ \\
\hline 18. & $30 / \mathrm{F}$ & 1465940 & ANT R LEG & $\begin{array}{l}\text { BIPHASIC- } \\
\text { PT, DP }\end{array}$ & NO & ANAEMIA & E. COLI & CIPRO, AMP & HEALED & $\begin{array}{c}12 \\
\text { WEEKS }\end{array}$ \\
\hline 19. & $23 / F$ & 1379606 & $\begin{array}{l}\text { R ANKLE } \\
\text { DORSUM }\end{array}$ & NORMAL & YES & NO & - & - & HEALED & $\begin{array}{c}8 \\
\text { WEEKS }\end{array}$ \\
\hline 20. & $40 / \mathrm{M}$ & 1460953 & $\begin{array}{l}\text { DORSUM OF R } \\
\text { FOOT }\end{array}$ & $\begin{array}{l}\text { MONOPHA } \\
\text { SIC-PT,DP }\end{array}$ & NO & HT & KLEB & CE, DOXY & HEALED & $\begin{array}{c}6 \\
\text { WEEKS } \\
\end{array}$ \\
\hline 21. & $50 / \mathrm{F}$ & 1416321 & $\begin{array}{l}\text { PLANTAR L } \\
\text { FOOT }\end{array}$ & $\begin{array}{l}\text { BIPHASIC } \\
\text { FLOW- } \\
\text { PT,DP }\end{array}$ & NO & CRF & KLEB & P, DOXY & HEALED & $\begin{array}{c}8 \\
\text { WEEKS }\end{array}$ \\
\hline 22. & $37 / F$ & 1396855 & $\begin{array}{l}\text { DORSUM R } \\
\text { FOOT }\end{array}$ & $\begin{array}{c}\text { BIPHASIC } \\
\text { FLOW- } \\
\text { PT,DP }\end{array}$ & NO & $\begin{array}{l}\text { HT/ANAE } \\
\text { MIA }\end{array}$ & E. COLI & GM,AMI & HEALED & $\begin{array}{c}12 \\
\text { WEEKS }\end{array}$ \\
\hline 23. & $31 / \mathrm{F}$ & 145948 & $\begin{array}{l}\text { DORSUM R } \\
\text { FOOT }\end{array}$ & $\begin{array}{l}\text { MONOPHA } \\
\text { SIC-DP }\end{array}$ & NO & NO & PSEUDO & $\mathrm{CE}, \mathrm{P}$ & HEALED & $\begin{array}{c}5 \\
\text { WEEKS } \\
\end{array}$ \\
\hline 24. & $50 / \mathrm{F}$ & 1475622 & $\begin{array}{l}\text { PLANTAR L } \\
\text { FOOT }\end{array}$ & $\begin{array}{l}\text { BIPHASIC- } \\
\text { DP } \\
\end{array}$ & YES & CAHD & E. COLI & CIPRO & HEALED & $\begin{array}{c}12 \\
\text { WEEKS }\end{array}$ \\
\hline 25. & $40 / \mathrm{F}$ & 1474135 & $\begin{array}{l}\text { DORSUM L } \\
\text { FOOT }\end{array}$ & $\begin{array}{c}\text { BIPHASIC } \\
\text { FLOW- } \\
\text { PT,DP }\end{array}$ & NO & HT & - & - & HEALED & $\begin{array}{c}9 \\
\text { WEEKS }\end{array}$ \\
\hline 26. & 49/M & 1410359 & LAT R LEG & $\begin{array}{l}\text { MONOPHA } \\
\text { SIC-PT,DP }\end{array}$ & YES & $\begin{array}{l}\text { CAHD/AN } \\
\text { AEMIA }\end{array}$ & STAPH & CE,AMP & HEALED & $\begin{array}{c}5 \\
\text { WEEKS }\end{array}$ \\
\hline 27. & $65 / M$ & 1459538 & $\begin{array}{l}\text { DORSUM R } \\
\text { FOOT }\end{array}$ & $\begin{array}{l}\text { BIPHASIC- } \\
\text { PT,DP }\end{array}$ & NO & $\mathrm{HT} / \mathrm{CRF}$ & E. COLI & GM,AMI & HEALED & $\begin{array}{c}7 \\
\text { WEEKS }\end{array}$ \\
\hline 28. & $55 / \mathrm{M}$ & 1465923 & ANT L LEG & $\begin{array}{l}\text { BIPHASIC } \\
\text { FLOW-DP }\end{array}$ & NO & CAHD/CRF & STREP & CE,P,CIPRO & HEALED & $\begin{array}{c}10 \\
\text { WEEKS }\end{array}$ \\
\hline 29. & $40 / \mathrm{F}$ & 1484503 & $\begin{array}{l}\text { DORSUM OF L } \\
\text { FOOT }\end{array}$ & NORMAL & NO & NO & - & - & HEALED & $\begin{array}{c}10 \\
\text { WEEKS }\end{array}$ \\
\hline 30. & $68 / \mathrm{M}$ & 1461280 & $\begin{array}{l}\text { DORSUM OF R } \\
\text { FOOT }\end{array}$ & $\begin{array}{l}\text { MONOPHA } \\
\text { SIC -PT,DP }\end{array}$ & NO & NO & - & - & HEALED & $\begin{array}{c}4 \\
\text { WEEKS } \\
\end{array}$ \\
\hline
\end{tabular}




\begin{tabular}{|c|c|c|c|c|c|c|c|c|c|c|}
\hline $\begin{array}{l}\text { Sl. } \\
\text { No. }\end{array}$ & $\begin{array}{l}\text { AGE } \\
\text { /SEX }\end{array}$ & IP NO. & ULCER SITE & $\begin{array}{c}\text { DOPPLER } \\
\text { STUDY }\end{array}$ & $\begin{array}{l}\text { NEURO- } \\
\text { PATHY }\end{array}$ & $\begin{array}{c}\text { RISK } \\
\text { FACTORS } \\
\end{array}$ & $\begin{array}{c}\text { PUS } \\
\text { CULTURE }\end{array}$ & $\begin{array}{c}\text { SENSITI } \\
\text { VITY }\end{array}$ & $\begin{array}{c}\text { HEALED/ } \\
\text { NOT }\end{array}$ & $\begin{array}{l}\text { DURA- } \\
\text { TION }\end{array}$ \\
\hline 1. & $65 / F$ & 1448391 & $\begin{array}{l}\text { DORSUM L } \\
\text { FOOT }\end{array}$ & $\begin{array}{c}\text { BIPHASIC } \\
\text { FLOW- } \\
\text { PA,PT,DP } \\
\end{array}$ & YES & NO & $\begin{array}{l}\text { ANAEROB } \\
\text { E }\end{array}$ & METRO & HEALED & 10 WEEKS \\
\hline 2. & $60 / F$ & 1388533 & $\begin{array}{c}\text { DORSUM OF } \\
\text { R FOOT } \\
\end{array}$ & $\begin{array}{l}\text { MONOPHASIC } \\
\text { FLOW-PT,DP }\end{array}$ & NO & CAHD/CRF & - & - & HEALED & 12 WEEKS \\
\hline 3. & $57 / \mathrm{M}$ & 1471602 & $\begin{array}{l}\text { PLANTAR R } \\
\text { FOOT }\end{array}$ & $\begin{array}{l}\text { MONOPHASIC } \\
\text { FLOW-DP }\end{array}$ & NO & $\mathrm{CRF} / \mathrm{HT}$ & KLEB & GM,AMI & $\begin{array}{c}\text { NOT } \\
\text { HEALED }\end{array}$ & \\
\hline 4. & $35 / F$ & 1433179 & $\begin{array}{l}\text { PLANTAR R } \\
\text { FOOT }\end{array}$ & $\begin{array}{l}\text { MONOPHASIC } \\
\text { FLOW-PT,DP }\end{array}$ & NO & $\mathrm{HT}$ & - & - & HEALED & 8 WEEKS \\
\hline 5. & $65 / M$ & 1475211 & $\begin{array}{l}\text { DORSUM R } \\
\text { FOOT }\end{array}$ & NORMAL & YES & NO & E. COLI & CIPRO & $\begin{array}{l}\text { NOTHEAL } \\
\text { ED }\end{array}$ & - \\
\hline 6. & $35 / \mathrm{M}$ & 1465412 & $\begin{array}{l}\text { PLANTAR R } \\
\text { FOOT }\end{array}$ & NORMAL & YES & CAHD/CRF & PSEUDO & AMI & HEALED & 9 WEEKS \\
\hline 7. & $49 / \mathrm{M}$ & 1459617 & $\begin{array}{l}\text { DORSUM L } \\
\text { FOOT }\end{array}$ & $\begin{array}{l}\text { MONOPHASIC } \\
\text { FLOW-DP,PT }\end{array}$ & YES & NO & STREP & $\mathrm{CE}$ & HEALED & 12 WEEKS \\
\hline 8. & $57 / F$ & 1444514 & $\begin{array}{l}\text { DORSUM R } \\
\text { FOOT }\end{array}$ & NORMAL & NO & NO & STREP & $\begin{array}{c}\text { CIPRO,D } \\
\text { OXY }\end{array}$ & HEALED & 11 WEEKS \\
\hline 9. & $20 / \mathrm{M}$ & 1466657 & $\begin{array}{l}\text { DORSUM L } \\
\text { FOOT }\end{array}$ & $\begin{array}{l}\text { BIPHASIC } \\
\text { FLOW-DP }\end{array}$ & YES & NO & E. COLI & AMI & HEALED & 12 WEEKS \\
\hline 10. & $50 / \mathrm{M}$ & 1476512 & $\begin{array}{l}\text { DORSUM R } \\
\text { FOOT }\end{array}$ & $\begin{array}{c}\text { MONOPHASIC } \\
\text { FLOW- } \\
\text { PA,DP,PT } \\
\end{array}$ & NO & CAHD/HT & KLEB & CE & HEALED & 10 WEEKS \\
\hline 11. & $53 / \mathrm{M}$ & 1461159 & $\begin{array}{l}\text { DORSUM L } \\
\text { FOOT }\end{array}$ & NORMAL & YES & $\mathrm{HT}$ & - & - & HEALED & 10 WEEKS \\
\hline 12. & $46 / \mathrm{M}$ & 1482650 & $\begin{array}{l}\text { DORSUM OF } \\
\text { L FOOT }\end{array}$ & $\begin{array}{l}\text { BIPHASIC } \\
\text { FLOW- } \\
\text { PA.PT,DP }\end{array}$ & NO & $\begin{array}{c}\text { ANAEMIA/ } \\
\text { HT }\end{array}$ & FUSIFORM & GM & $\begin{array}{l}\text { NOTHEAL } \\
\text { ED }\end{array}$ & - \\
\hline 13. & $46 / F$ & 1422813 & $\begin{array}{l}\text { DORSUM L } \\
\text { FOOT }\end{array}$ & $\begin{array}{l}\text { MONOPHASIC } \\
\text { FLOW-PA,DP }\end{array}$ & NO & CRF & $\begin{array}{c}\text { E. } \\
\text { COLI,STAP } \\
\text { H }\end{array}$ & $\begin{array}{l}\text { CE,CIPR } \\
0\end{array}$ & HEALED & 10 WEEKS \\
\hline 14. & $30 / \mathrm{F}$ & 1462334 & $\begin{array}{l}\text { PLANTAR L } \\
\text { FOOT }\end{array}$ & $\begin{array}{c}\text { MONOPHASIC } \\
\text { FLOW-PT,DP }\end{array}$ & YES & HT & KLEB & $P$ & HEALED & 13 WEEKS \\
\hline 15. & $29 / F$ & 1422837 & $\begin{array}{l}\text { DORSUM R } \\
\text { FOOT }\end{array}$ & $\begin{array}{c}\text { BIPHASIC } \\
\text { FLOW-PT,DP }\end{array}$ & NO & ANAEMIA & E. COLI & GM,AMI & HEALED & 7 WEEKS \\
\hline 16. & $45 / \mathrm{M}$ & 1464625 & L THIGH & NORMAL & YES & NO & - & - & HEALED & 6 WEEKS \\
\hline 17. & $61 / M$ & 1476330 & $\begin{array}{l}\text { DORSUM OF } \\
\text { L FOOT }\end{array}$ & $\begin{array}{l}\text { MONOPHASIC } \\
\text { FLOW-DP }\end{array}$ & NO & $\begin{array}{c}\text { CRF/HT/C } \\
\text { AHD }\end{array}$ & $\begin{array}{l}\text { ANAEROB } \\
\text { E }\end{array}$ & METRO & HEALED & 9 WEEKS \\
\hline 18. & $54 / \mathrm{M}$ & 1483944 & $\begin{array}{l}\text { DORSUM L } \\
\text { FOOT }\end{array}$ & $\begin{array}{c}\text { MONOPHASIC } \\
\text { FLOW- PT,DP }\end{array}$ & NO & CRF & E. COLI & $\begin{array}{c}\text { CE,CIPR } \\
0 \\
\end{array}$ & $\begin{array}{c}\text { NONHEALI } \\
\text { NG }\end{array}$ & \\
\hline 19. & $55 / \mathrm{M}$ & 14675164 & $\begin{array}{l}\text { PLANTAR L } \\
\text { FOOT }\end{array}$ & $\begin{array}{l}\text { BIPHASIC } \\
\text { FLOW-DP }\end{array}$ & NO & CRF/CAHD & STREP & CE,AMI & $\begin{array}{c}\text { NON } \\
\text { HEALING }\end{array}$ & \\
\hline 20. & $46 / \mathrm{F}$ & 1385758 & $\begin{array}{l}\text { DORSUM OF } \\
\text { R FOOT }\end{array}$ & $\begin{array}{l}\text { MONOPHASIC } \\
\text { FLOW-PT,DP }\end{array}$ & YES & ANAEMIA & - & - & HEALED & 14 WEEKS \\
\hline 21. & $40 / \mathrm{F}$ & 1479293 & $\begin{array}{l}\text { DORSUM OF } \\
\text { L FOOT }\end{array}$ & NORMAL & YES & NO & PSEUDO & $\begin{array}{c}\text { AMI,AM } \\
\mathrm{P} \\
\end{array}$ & HEALED & 7 WEEKS \\
\hline 22. & $35 / F$ & 1479293 & $\begin{array}{c}\text { DORSUM OF } \\
\text { L FOOT }\end{array}$ & NORMAL & YES & NO & FUSIFORM & $\begin{array}{c}\text { GM,SEPT } \\
\text { RAN }\end{array}$ & $\begin{array}{c}\text { NON- } \\
\text { HEALING }\end{array}$ & - \\
\hline 23. & $40 / \mathrm{M}$ & 1466951 & $\begin{array}{l}\text { DORSUM R } \\
\text { FOOT }\end{array}$ & $\begin{array}{l}\text { MONOPHASIC } \\
\text { FLOW-PT,DP }\end{array}$ & NO & HT & STREP & $P$ & HEALED & 7 WEEKS \\
\hline 24. & $60 / \mathrm{M}$ & 1469012 & ANT R LEG & NORMAL & YES & NO & - & - & HEALED & 8 WEEKS \\
\hline 25. & $50 / \mathrm{F}$ & 1444341 & $\begin{array}{l}\text { DORSUM OF } \\
\text { R FOOT }\end{array}$ & $\begin{array}{c}\text { MONOPHASIC } \\
\text { FLOW-PA, PT, } \\
\text { DP }\end{array}$ & NO & $\begin{array}{l}\text { CAHD/AN } \\
\text { AEMIA }\end{array}$ & $\begin{array}{c}\text { E. } \\
\text { COLI,KLEB }\end{array}$ & $\begin{array}{l}\text { CE,CIPR } \\
0\end{array}$ & $\begin{array}{c}\text { NON- } \\
\text { HEALING }\end{array}$ & - \\
\hline 26. & $65 / \mathrm{M}$ & 1470373 & $\begin{array}{c}\text { DORSUM OF } \\
\text { R FOOT }\end{array}$ & $\begin{array}{l}\text { MONOPHASIC } \\
\text { FLOW-PT, DP }\end{array}$ & NO & ANAEMIA & E. COLI & $\mathrm{P}, \mathrm{GM}$ & $\begin{array}{c}\text { NON- } \\
\text { HEALING }\end{array}$ & \\
\hline 27. & $42 / \mathrm{M}$ & 14722118 & $\begin{array}{l}\text { DORSUM OF } \\
\text { L FOOT }\end{array}$ & $\begin{array}{c}\text { BIPHASIC } \\
\text { FLOW-PA, PT, } \\
\text { DP }\end{array}$ & NO & ANAEMIA & $\begin{array}{l}\text { ANAEROB } \\
\text { E }\end{array}$ & METRO & $\begin{array}{c}\text { NON- } \\
\text { HEALING }\end{array}$ & - \\
\hline 28. & $57 / \mathrm{M}$ & 1468143 & $\begin{array}{l}\text { DORSUM OF } \\
\text { L FOOT }\end{array}$ & $\begin{array}{l}\text { MONOPHASIC } \\
\text { FLOW-PT,DP }\end{array}$ & YES & CAHD,HT & STAPH & $\begin{array}{c}\text { CIPRO,A } \\
\text { MI }\end{array}$ & $\begin{array}{c}\text { NON- } \\
\text { HEALING }\end{array}$ & \\
\hline 29. & $55 / F$ & 1479601 & $\begin{array}{l}\text { DORSUM OF } \\
\text { R FOOT }\end{array}$ & NORMAL & YES & NO & PSEUDO & CIPRO & HEALED & 8 WEEKS \\
\hline 30. & $35 / \mathrm{M}$ & 1462911 & $\begin{array}{l}\text { DORSUM OF } \\
\text { R FOOT }\end{array}$ & $\begin{array}{c}\text { BIPHASIC } \\
\text { FLOW-PT, DP }\end{array}$ & NO & NO & KLEB & AMP, GM & HEALED & 7 WEEKS \\
\hline
\end{tabular}

\section{REFERENCES}

1. en.wikipedia.org/wiki/wound_healing.

2. www.ncbi.nlm.nih.gov/pubmed/17496352.

3. Hall K. The nobel prize in physiology or medicine 1986presentation speech. The nobel foundation retrieved 2009-04-24, 1986.
4. Wong WR, Lam E, Huang RC, et al. Application, and efficient large scale production of recombinant human epidermal growth factor. Biotechnol Genet Eng Rev 2001;18:51-71.

DOI: $10.1080 / 02648725.2001 .10648008$. 
5. Dreux AC, Lamb DJ, Modjtahedi H, et al. The epidermal growth factor receptors and their family of ligands: their putative role in atherogenesis. Atherosclerosis 2006;186 (1):38-53.

6. Man Wo Tsang, Wan Keung R Wong, Chi Sang Hung. Human epidermal growth factor enhances healing of diabetic foot ulcers. Diabetes care 2003;26:1856-61.

7. Ulrich Marti, Susan jo burwen, Albert L Jones. Biological effects of epidermal growth factor, with the emphasis on the gastrointestinal tract and liver: an update. Article in hepatology 1989;9(1):126-38.

8. Elizabeth D Hay, Thomas J King, John Papaconstatinou. Macromolecules regulating growth and developmental. The $30^{\text {th }}$ symposium was held at seattle, wahington, june 1971;17-19.
9. Carpenter G, Cohen S. Epidermal growth factor. The journal of biological chemistry 1990;265(14):7709-12.

10. Herbst RS. Review of epidermal growth factor receptor biology. International journal of radiation oncology, biology, physics 2004;59(2):21-6.

11. Graham Carpenter. The regulation of cell proliferation: advances in the biology and mechanism of action of epidermal growth factor. Journal of investigative dermatology 1978;71(5):283-8. DOI:10.1111/1523-1747.ep12529177. 